\section{Australian Journal of \\ Crop Science}

AJCS 11(05):496-505 (2017)

doi: 10.21475/ajcs.17.11.05.p260
AJCS

ISSN:1835-2707

\title{
Field application of plant resistance inducers (PRIs) to control important root rot diseases and improvement growth and yield of green bean (Phaseolus vulgaris $\mathbf{L}$.)
}

\author{
Riad Sedki Riad El-Mohamedy ${ }^{1}$, Mohamed Reda Shafeek ${ }^{2}$, Emad El-Din Hassanein Abd El-Samad ${ }^{2 *}$, \\ Dina Mohamed Salama², Fatma Ahmed Rizk²
}

\author{
${ }^{1}$ Plant Pathology Department, National Research Centre, 33 El-Buhouth St., Dokki, Giza, Egypt, Postal Code: \\ 12622. \\ ${ }^{2}$ Vegetable Research Department, National Research Centre, 33 El-Buhouth St., Dokki, Giza, Egypt, Postal \\ Code: 12622.
}

*Corresponding author: emadhassanein@ hotmail.com

\begin{abstract}
The efficiency of plant resistance inducers (PRIs) i.e. chitosan (CH), salicylic acid (SA) and humic acid (HA) in controlling Fusarium solani and Rhizoctonia solani the causal agents of root rot diseases under in vitro and on growth and yield of green bean cv. Giza 3 were evaluated. In vitro treatments of $\mathrm{CH} 1.0 \mathrm{~g} / \mathrm{l}, \mathrm{CH} 1.0 \mathrm{~g} / \mathrm{l}+\mathrm{HA} 5.0 \%$ and $\mathrm{CH} 0.5 \mathrm{~g} / \mathrm{l}+\mathrm{SA} 2.5 \%$, as sterilized PDA medium addition, caused the highest reduction in mycelia growth and spore germination of $F$. solani and $R$. solani on incubated plates compared to control plates (medium free of inducers). Under greenhouse conditions, combined treatments of PRIs were superior than single treatments, while, $\mathrm{CH} 1.0 \mathrm{~g} / \mathrm{l}+\mathrm{SA} 5 \%$ or HA $5.0 \%$ treatments were more effective than single treatments in controlling of Rhizoctonia root rot. These treatments caused disease reduction reached to 70.4 and $67.4 \%$ of pre-emergence stage after 10 days of seed sowing, and 62.8 and $60.4 \%$ of post-emergence stage after 40 days of seed sowing. Meanwhile, the highest values of survival plants 79.4 and $77.8 \%$ were recorded with the same treatments. Under field conditions, the obtained results indicated that soaking bean seeds in $\mathrm{CH} 1.0 \mathrm{~g} / \mathrm{l}+\mathrm{SA} 5 \%$ followed by foliar application with half of this concentrations $\mathrm{CH} 0.5 \mathrm{~g} / \mathrm{l}+$ SA 2.5\%, (for 3 times in 7 days interval starting from the second true leaf stage), resulted in the higher percentages of reduction of damping-off and root rot incidence 68.2, 63.8, 70.0\% and 67.4, 63.3, 69.6\% after 10, 40 and 60 days during both seasons, respectively, compared with other treatments. Furthermore, such treatment was superior than commercial fungicide seed treatment in controlling of green bean root rot, where, fungicide treatment reduced damping-off and root rot incidence after 10, 40 and 60 days up to $64.6,60.4,64.0 \%$ and $64.0,59.6,63.4 \%$ during both seasons. Combined treatments of PRIs and commercial fungicide treatment resulted in increasing of vegetative growth characters, yield and quality of green bean pods compared to other treatments during two successive seasons. It could be suggested that using plant resistance inducers in comparison with fungicides, might be considered as an eco-friendly, safe, cheap and easily applied method for controlling such soil-borne plant pathogens considering the avoidance of environmental pollution and the side effects of fungicides application.
\end{abstract}

Keywords: Green bean, Plant resistance inducers, Chitosan, Humic acid, Salicylic acid, Root rot diseases, Vegetative growth, pods yield and quality.

Abbreviations: PRIs_Plant resistance inducers, CH_chitosan, SA_salicylic acid and HA_humic acid.

\section{Introduction}

Green bean (Phaseolus vulgaris L.) is one of the most important vegetable food legumes in Egypt. It is consumed either as green pods or dry seeds. It represents a cheap source of calcium, proteins and some amino acids especially lysine in human diets. Recently, it is highly demanded for exporting to the European market, the exported amount reached to 15000 tons in 2015 according to agriculture statistics.

Green bean diseases are the major constraints to production, reducing yield and seed quality. Bean is attacked by certain soil borne fungi causing root rot diseases. The main pathogens responsible for root rot disease are Fusarium solani and Rhizoctonia solani, it is a serious disease for green bean plants (Abdel-Kader, 1997; El-Mougy et al., 2007).

Lately, a considerable concern to reach or develop alternative applications of fungicidal seed treatments for controlling such plant diseases has been paid. Systemic acquired resistance
(SAR) or induction of plants resistance against pathogen is a promising approach for controlling plant diseases. Therefore, induction of plant resistance to overcome pathogen infection is an effective way for controlling plant diseases. The phenomenon of plant resistance to pathogens can be enhanced by the application of various abiotic agent (chemical inducers or elicitors), caused an induction of systemic resistance in plants (El-Khallal, 2007; Akram and Anjum, 2011; Abdel-Monaim et al., 2012). Conversely, application of such inducer resistance chemicals under field conditions have been proved to increase vegetative growth, yield and quality of many vegetable crops (El-Mougy et al., 2007; Abdel-Mawgoud et al., 2010; Shehata et al., 2012; Bakeer, 2014; Abd El-Gawad and Bondok, 2015; ElMohamedy et al., 2015). 
Chitosan $(\mathrm{CH})$ is a natural carbohydrate polymer derived from crustaceous shells such as crabs and shrimps. It is an eco-friendly, nontoxic, bioactive agent has become a useful appreciated compound due to its fungicidal effects and elicitation of natural defense mechanisms in plant tissues (Terry and Joyce, 2004). In addition, chitosan treatment has been shown to stimulate plant growth (Kim, 2005). Shehata et al. (2012) concluded that foliar application with chitosan at rate of $4 \mathrm{ml} / \mathrm{l}$ gave the highest vegetative growth, yield and quality of cucumber plants.

Salicylic acid (SA) is a phenolic compound that acts as a key regulator of the endogenous signaling network in plants under abiotic and biotic stresses. It plays a key role in the plant resistance to pathogens by inducing the production of pathogenesis-related proteins (PRPs). Also salicylic acid was responsible for the accumulation of phyto-alexins in viable plant tissues (Anderson, 1988).

Soaking sesame seeds in salicylic acid at rate of $5 \mathrm{mM}$ for $24 \mathrm{~h}$ before sowing followed by foliar application after 15 days from sowing resulted in the best control against $F$. oxysporum compared to fungicide Benlate (Abdou et al., 2001). Shahda (2000) stated that using benzoic acid, salicylic acid and ascorbic acid significantly reduced damping-off, the reduction in disease incidence ranged from $36-74 \%$ and exhibited growth promoting effect. The antioxidants used were superior to fungicide Rizolex-T either in controlling the disease or in promoting the plant growth. Abd El-Gawad and Bondok (2015) suggested that foliar application of $\mathrm{CH}$ plus SA could be used to reduce the negative effect of viral infection of TMV and improve the growth, mineral contents and yield of tomato plants grown under open field conditions. Humic acid (HA) is a principal component of humic substances, which are the major organic constituents of soil (humus). It is a complex mixture of many different acids containing carboxyl and phenolate groups. Humic acids can form complexes with ions that are commonly found in the environment creating humic colloids. It could be applied successfully in many areas of plant production as a plant growth stimulant or soil conditioner or inducing natural resistance against plant diseases, whereas, several reports indicated the efficiency of HA in reducing some plant diseases (Scheuerell and Mahaffee, 2004), stimulation plant growth through increased cell division, as well as optimized uptake of nutrients and water and stimulated the soil microorganisms (Chen et al., 2004).

Application of humic acid enhances plant growth, nutrient uptake, plant yield and quality in a number of plant species (Zaky et al., 2006; Yildirim, 2007; Karakurt et al., 2009). In the same respect, application of humic acid at rate of $4 \mathrm{~g} / \mathrm{l}$, led significantly to increase vegetative growth as well as total yield. Moreover, increasing the percentage of nitrogen and protein contents as well as Fe content in broad bean seeds (Shafeek et al., 2013). Also, Abdel-Monaim et al. (2011) noted that combined treatment between benzothiadiazole and humic acid might be used commercially as a safety method for controlling soybean diseases under open field conditions. Several reports indicated the efficiency of inducer resistance chemicals in reducing some plant diseases. The use of such chemicals inducers, would permit a reduction in the use of agrochemicals such fungicides (Abdel-Kareem, 2007; Yigit and Dikilitas, 2008; El-Mohamedy and Ahmed, 2009; Bakeer, 2014).

The objective of the current study was to evaluate the efficiency of seed soaking and foliar applications of some chemicals for plant resistance inducer as an alternative applications of fungicidal in comparison with commercial fungicide (Rizolex-T) against root rot diseases and on vegetative growth, yield and quality of green bean grown under open field conditions.

\section{Results and Discussion}

\section{Effect of plant resistance inducers (PRIs) on linear growth of $F$. solani and $R$. solani}

Plant resistance inducers (PRIs) i.e. chitosan $(\mathrm{CH})$, salicylic acid (SA) and humic acid (HA) alone or in combined treatments at different concentrations were tested for their inhibitory effect on linear growth of $F$. solani and $R$. solani in vitro. Results in Table (1) indicated that all tested PRIs had an inhibitor effect on linear growth of $F$. solani and $R$. solani in varying degrees, except salicylic acid treatment at rate of $1.25 \%$ and humic acid at all tested rates as well as control treatment. The inhibitory effect increased by increasing PRIs concentrations or in another means, the minimum linear growth was recorded at the maximum concentrations of PRIs. Meanwhile, complete reduction in linear growth was observed with chitosan at $2.0 \mathrm{~g} / \mathrm{l}$ and $\mathrm{CH}+\mathrm{SA}$ at $1.0 \mathrm{~g} / \mathrm{l}+$ $5.0 \%$ treatments as shown in Fig. 1. In general, the highest reduction in mycelia growth of both fungus was recorded at concentrations of $2.0 \mathrm{~g} / \mathrm{l}, 1.0 \mathrm{~g} / \mathrm{l}+5.0 \%$ and $1.0 \mathrm{~g} / \mathrm{l}+5 \%$ of chitosan, chitosan + salicylic acid and chitosan + humic acid treatments, respectively. They reduced the fungal linear growth down to 100,100 and $90 \%$ for $F$. solani and 100, 100 and $88.9 \%$ for $R$. solani.

Results are in agreement with finding of many authors, they mentioned that many chemical plant resistance inducers recorded the highest inhibition of growth of many pathogenic fungi (Abdel-Monaim, 2013; Bakeer, 2014). The inhibitory effect produced by SA in the present study almost agreed with that found by Hilal et al. (2006) who found that salicylic acid completely prevented the mycelia growth of Sclerotinia sclerotiorum. Also Abdel-Monaim et al. (2012) showed that $\mathrm{SA}$ at $200 \mathrm{ppm}$ decreased liner growth of $F$. oxysorum, $F$. solani and R. solani from $90 \mathrm{~mm}$ in check treatment to 54.93 , 48.60 and $65.45 \mathrm{~mm}$, respectively.

\section{Control of root rot disease of green bean under greenhouse conditions}

Efficiency of some chemical plant resistance inducers i.e. chitosan (CH), salicylic acid (SA) and humic acid (HA) alone or in combined treatments at different concentrations and seed dressing with commercial fungicide (Rizolex-T) as a comparison treatment were applied to control the main root rot pathogens Fusarium solani and Rhizoctonia solani of green bean plants under artificially infested soil in greenhouse.

Data in Table 2 visibly showed that all applied PRIs treatments significantly decreased Rhizoctonia root rot at pre and post-emergence stages if compared with control treatment. Combined treatments of chitosan $1.0 \mathrm{~g} / \mathrm{l}+\mathrm{SA}$ or $\mathrm{HA}$ at rate of $5.0 \%$ were more effective than single treatments for controlling of Rhizoctonia root rot. As, these treatments caused disease reduction percentages reached to 70.4 and $67.4 \%$ of pre-emergence after 10 days of seeding and 62.8 and $60.4 \%$ of post- emergence stage after 40 days of seeding, as well as the highest percentages of survival plants 79.4 and $77.8 \%$ were recorded with the same treatments. Meanwhile, combination treatments between chitosan $1.0 \mathrm{~g} / \mathrm{l}$ $+\mathrm{SA}$ or HA at rate of $2.5 \%$ caused a considerable effect, they reduced root rot incidence by 60.2 and $59.2 \%$ at preemergence and by 59.8 and $58.2 \%$ at post-emergence and gave 76.1 and $76.2 \%$ for the percentage of survival plants. 
Table 1. Linear growth and percentage of linear growth reduction of Fusarium solani and Rhizoctonia solani in vitro as affected by different concentrations of plant resistance inducers.

\begin{tabular}{|c|c|c|c|c|c|}
\hline \multirow[b]{2}{*}{$\begin{array}{l}\text { Plant } \\
\text { resistance } \\
\text { inducers }\end{array}$} & \multirow[b]{2}{*}{$\begin{array}{l}\text { Treatments/ } \\
\text { concentrations }\end{array}$} & \multicolumn{2}{|c|}{$F$. solani } & \multicolumn{2}{|c|}{$R$. solani } \\
\hline & & $\begin{array}{l}\text { Linear } \\
\text { growth } \\
(\mathrm{mm})\end{array}$ & $\begin{array}{l}\text { Reduction } \\
\text { of linear } \\
\text { growth\% }\end{array}$ & $\begin{array}{l}\text { Linear growth } \\
\quad(\mathrm{mm})\end{array}$ & $\begin{array}{l}\text { Reduction } \\
\text { of linear } \\
\text { growth\% }\end{array}$ \\
\hline \multirow{4}{*}{$\begin{array}{l}\text { Chitosan } \\
(\mathrm{CH})\end{array}$} & $0.25 \mathrm{~g} / 1$ & $64 \mathrm{a}$ & 28.9 & $70 \mathrm{a}$ & 22.2 \\
\hline & $0.5 \mathrm{~g} / 1$ & $35 \mathrm{c}$ & 61.1 & $38 \mathrm{c}$ & 57.8 \\
\hline & $1.0 \mathrm{~g} / 1$ & $10 \mathrm{~d}$ & 88.9 & $12 \mathrm{~d}$ & 86.7 \\
\hline & $2.0 \mathrm{~g} / 1$ & $0.0 \mathrm{~d}$ & 100 & $0.0 \mathrm{~d}$ & 100 \\
\hline \multirow{3}{*}{$\begin{array}{l}\text { Salicylic acid } \\
\text { (SA) }\end{array}$} & $1.25 \%$ & $90 \mathrm{a}$ & 0.0 & $90 \mathrm{a}$ & 0.0 \\
\hline & $2.5 \%$ & $62 \mathrm{a}$ & 31.1 & $66 \mathrm{a}$ & 26.7 \\
\hline & $5.0 \%$ & $40 \mathrm{~b}$ & 44.4 & $50 \mathrm{~b}$ & 44.4 \\
\hline \multirow{3}{*}{$\begin{array}{l}\text { Humic acid } \\
\text { (HA) }\end{array}$} & $1.25 \%$ & $90 a$ & 0.0 & $90 \mathrm{a}$ & 0.0 \\
\hline & $2.5 \%$ & $90 \mathrm{a}$ & 0.0 & $90 \mathrm{a}$ & 0.0 \\
\hline & $5.0 \%$ & $90 \mathrm{a}$ & 0.0 & $90 \mathrm{a}$ & 0.0 \\
\hline $\mathrm{CH}+\mathrm{SA}$ & $0.5 \mathrm{~g} / 1+2.5 \%$ & $17 \mathrm{~d}$ & 81.1 & $26 \mathrm{~d}$ & 71.1 \\
\hline $\mathrm{CH}+\mathrm{SA}$ & $1.0 \mathrm{~g} / 1+5.0 \%$ & $0.0 \mathrm{~d}$ & 100 & $0.0 \mathrm{~d}$ & 100 \\
\hline $\mathrm{CH}+\mathrm{HA}$ & $0.5 \mathrm{~g} / 1+2.5 \%$ & $30 \mathrm{c}$ & 66.7 & $30 \mathrm{c}$ & 66.7 \\
\hline $\mathrm{CH}+\mathrm{HA}$ & $1.0 \mathrm{~g} / 1+5.0 \%$ & $9.0 \mathrm{~d}$ & 90.0 & $10 \mathrm{~d}$ & 88.9 \\
\hline $\mathrm{SA}+\mathrm{HA}$ & $2.5 \%+2.5 \%$ & $55 \mathrm{~b}$ & 38.9 & $56 \mathrm{~b}$ & 37.8 \\
\hline $\mathrm{SA}+\mathrm{HA}$ & $5.0 \%+5.0 \%$ & $26 \mathrm{c}$ & 71.1 & $34 \mathrm{c}$ & 62.2 \\
\hline Control & & $90.0 \mathrm{a}$ & 0.0 & $90.0 \mathrm{a}$ & 0.0 \\
\hline
\end{tabular}

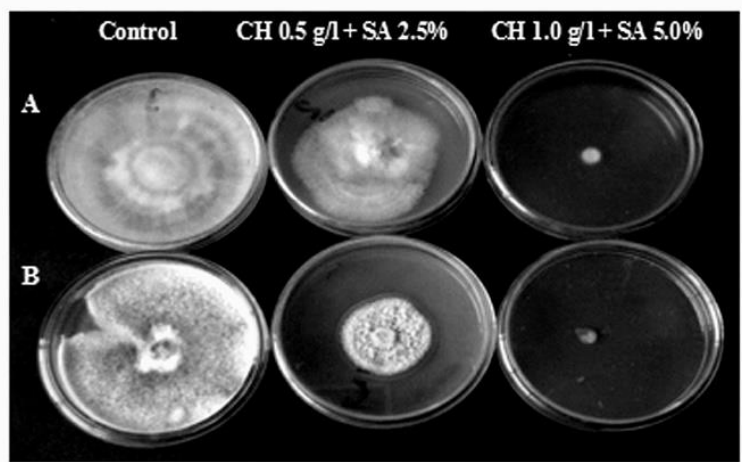

Fig 1. Linear growth of Fusarium solani (A) and Rhizoctonia solani (B) as affected by different concentration of formulated plant resistance inducers.

Table 2. Effect of chitosan, salicylic acid and humic acid as chemical plant resistance inducers in single or combined treatments on damping-off and root rot disease incidence of green bean plants grown in infested soil with Rhizoctonia solani under greenhouse conditions.

\begin{tabular}{|c|c|c|c|c|c|c|c|}
\hline \multirow{2}{*}{$\begin{array}{l}\text { Plant resistance } \\
\text { inducers }\end{array}$} & \multicolumn{2}{|c|}{ Treatments/concentrations } & \multicolumn{2}{|c|}{$\begin{array}{c}\text { Pre-emergence damping } \\
\text { off after } 10 \text { days }\end{array}$} & \multicolumn{2}{|c|}{$\begin{array}{l}\text { Pre-emergence damping off } \\
\text { after } 40 \text { days }\end{array}$} & \multirow{2}{*}{$\begin{array}{c}\text { Survival } \\
\text { plants } \\
\%\end{array}$} \\
\hline & $\begin{array}{l}\text { Seed } \\
\text { soaking }\end{array}$ & $\begin{array}{l}\text { Foliar } \\
\text { spraying }\end{array}$ & Infection & $\begin{array}{c}\text { Disease } \\
\text { reduction } \%\end{array}$ & Infection & $\begin{array}{c}\text { Disease } \\
\text { reduction } \%\end{array}$ & \\
\hline Chitosan $(\mathrm{CH})$ & $1.0 \mathrm{~g} / \mathrm{l}$ & $0.5 \mathrm{~g} / \mathrm{l}$ & $7.4 \mathrm{~d}$ & 58.8 & $17.8 \mathrm{~b}$ & 54.4 & 76.7 \\
\hline Salicylic acid & $2.5 \%$ & $1.25 \%$ & $16.4 \mathrm{~b}$ & 41.6 & $19.2 \mathrm{~b}$ & 40.4 & 64.6 \\
\hline (SA) & $5 \%$ & $2.5 \%$ & $12.6 \mathrm{c}$ & 55.1 & $15.8 \mathrm{c}$ & 51.2 & 71.6 \\
\hline Humic acid & $2.5 \%$ & $1.25 \%$ & $16.8 \mathrm{~b}$ & 40.2 & $19.2 \mathrm{~b}$ & 40.8 & 64.6 \\
\hline (HA) & $5 \%$ & $2.5 \%$ & $13.8 \mathrm{c}$ & 50.4 & $17.4 \mathrm{~b}$ & 45.4 & 68.4 \\
\hline $\mathrm{CH}+\mathrm{SA}$ & $1.0 \mathrm{~g} / \mathrm{l}+2.5 \%$ & $0.5 \mathrm{~g} / \mathrm{l}+1.25 \%$ & $11.2 \mathrm{c}$ & 60.2 & $13.0 \mathrm{~d}$ & 59.8 & 76.1 \\
\hline $\mathrm{CH}+\mathrm{SA}$ & $1.0 \mathrm{~g} / 1+5 \%$ & $0.5 \mathrm{~g} / 1+2.5 \%$ & $8.2 \mathrm{~d}$ & 70.4 & $12.0 \mathrm{~d}$ & 62.8 & 79.4 \\
\hline $\mathrm{CH}+\mathrm{HA}$ & $1.0 \mathrm{~g} / 1+2.5 \%$ & $0.5 \mathrm{~g} / 1+1.25 \%$ & $11.4 \mathrm{c}$ & 59.2 & $13.4 \mathrm{~d}$ & 58.2 & 76.2 \\
\hline $\mathrm{CH}+\mathrm{HA}$ & $1.0 \mathrm{~g} / \mathrm{l}+5 \%$ & $0.5 \mathrm{~g} / \mathrm{l}+2.5 \%$ & $9.2 \mathrm{~d}$ & 67.4 & $12.8 \mathrm{~d}$ & 60.4 & 77.8 \\
\hline $\mathrm{SA}+\mathrm{HA}$ & $2.5+2.5 \%$ & $1.25+1.25 \%$ & $12.0 \mathrm{c}$ & 56.8 & $17.8 \mathrm{~b}$ & 54.2 & 70.2 \\
\hline $\mathrm{SA}+\mathrm{HA}$ & $5+5 \%$ & $2.5+2.5 \%$ & $12.6 \mathrm{c}$ & 55.2 & $15.4 \mathrm{c}$ & 51.0 & 72.0 \\
\hline \multicolumn{3}{|c|}{ Fungicide (Rhizolex-T 3g/kg seeds) } & $8.2 \mathrm{~d}$ & 70.8 & $11.4 \mathrm{~d}$ & 64.8 & 80.3 \\
\hline \multicolumn{3}{|c|}{ Control } & $28.0 \mathrm{a}$ & 0.0 & $32.4 \mathrm{a}$ & 0.0 & 27.6 \\
\hline
\end{tabular}


Table 3. Effect of chitosan, salicylic acid and humic acid as chemical plant resistance inducers in single or combined treatments on damping-off and root rot disease incidence of green bean plants grown in infested soil with Fusarium solani under greenhouse conditions.

\begin{tabular}{|c|c|c|c|c|c|c|c|}
\hline \multirow{2}{*}{$\begin{array}{l}\text { Plant resistance } \\
\text { inducers }\end{array}$} & \multicolumn{2}{|c|}{ Treatments/concentrations } & \multicolumn{2}{|c|}{$\begin{array}{c}\text { Pre-emergence damping } \\
\text { off after } 10 \text { days }\end{array}$} & \multicolumn{2}{|c|}{$\begin{array}{c}\text { Pre-emergence damping } \\
\text { off after } 40 \text { days }\end{array}$} & \multirow{2}{*}{$\begin{array}{c}\text { Survival } \\
\text { plants } \\
\%\end{array}$} \\
\hline & $\begin{array}{l}\text { Seed } \\
\text { soaking }\end{array}$ & $\begin{array}{l}\text { Foliar } \\
\text { spraying }\end{array}$ & Infection & $\begin{array}{c}\text { Disease } \\
\text { reduction } \%\end{array}$ & Infection & $\begin{array}{c}\text { Disease } \\
\text { reduction } \%\end{array}$ & \\
\hline Chitosan $(\mathrm{CH})$ & $1.0 \mathrm{~g} / \mathrm{l}$ & $0.5 \mathrm{~g} / \mathrm{l}$ & $11.4 \mathrm{c}$ & 57.0 & $18.8 \mathrm{~d}$ & 52.6 & 67.7 \\
\hline \multirow{2}{*}{$\begin{array}{l}\text { Salicylic acid } \\
\text { (SA) }\end{array}$} & $2.5 \%$ & $1.25 \%$ & $13.8 \mathrm{~b}$ & 39.8 & $24.0 \mathrm{~b}$ & 34.8 & 55.2 \\
\hline & $5 \%$ & $2.5 \%$ & $12.0 \mathrm{bc}$ & 48.1 & $19.8 \mathrm{c}$ & 46.4 & 62.5 \\
\hline \multirow{2}{*}{$\begin{array}{l}\text { Humic acid } \\
\text { (HA) }\end{array}$} & $2.5 \%$ & $1.25 \%$ & $14.4 \mathrm{~b}$ & 37.8 & $23.2 \mathrm{~b}$ & 36.6 & 55.3 \\
\hline & $5 \%$ & $2.5 \%$ & $12.2 \mathrm{c}$ & 47.2 & $20.0 \mathrm{c}$ & 45.2 & 61.7 \\
\hline $\mathrm{CH}+\mathrm{SA}$ & $1.0 \mathrm{~g} / 1+2.5 \%$ & $0.5 \mathrm{~g} / 1+1.25 \%$ & $9.0 \mathrm{~d}$ & 61.2 & $16.8 \mathrm{~d}$ & 54.4 & 69.7 \\
\hline $\mathrm{CH}+\mathrm{SA}$ & $1.0 \mathrm{~g} / 1+5 \%$ & $0.5 \mathrm{~g} / \mathrm{l}+2.5 \%$ & $8.0 \mathrm{~d}$ & 65.4 & $14.4 \mathrm{e}$ & 60.7 & 73.5 \\
\hline $\mathrm{CH}+\mathrm{HA}$ & $1.0 \mathrm{~g} / 1+2.5 \%$ & $0.5 \mathrm{~g} / \mathrm{l}+1.25 \%$ & $9.4 \mathrm{~d}$ & 59.6 & $17.8 \mathrm{~d}$ & 52.2 & 68.2 \\
\hline $\mathrm{CH}+\mathrm{HA}$ & $1.0 \mathrm{~g} / 1+5 \%$ & $0.5 \mathrm{~g} / \mathrm{l}+2.5 \%$ & $8.4 \mathrm{~d}$ & 63.5 & $15.4 \mathrm{e}$ & 57.8 & 71.7 \\
\hline $\mathrm{SA}+\mathrm{HA}$ & $2.5+2.5 \%$ & $1.25+1.25 \%$ & $10.2 \mathrm{c}$ & 56.4 & $17.2 \mathrm{~d}$ & 53.0 & 72.6 \\
\hline $\mathrm{SA}+\mathrm{HA}$ & $5+5 \%$ & $2.5+2.5 \%$ & $10.4 \mathrm{c}$ & 53.4 & $17.8 \mathrm{~d}$ & 51.8 & 71.8 \\
\hline \multicolumn{3}{|c|}{ Fungicide (Rhizolex-T 3g/kg seeds) } & $8.0 \mathrm{~d}$ & 65.6 & $13.2 \mathrm{e}$ & 64.2 & 75.0 \\
\hline \multicolumn{3}{|c|}{ Control } & $23.2 \mathrm{a}$ & 0.0 & $36.8 \mathrm{a}$ & 0.0 & 28.9 \\
\hline
\end{tabular}

Commercial fungicide treatment reduced disease incidence by 70.8 and $64.8 \%$ at pre and post-emergence stages, respectively. Single treatments of $\mathrm{CH}, \mathrm{SA}$ and $\mathrm{HA}$ at the highest concentrations resulted in disease reduction up to $50 \%$ at both pre and post-emergence stages. Meanwhile at the lowest concentrations of these single treatments showed less effect in controlling of root rot at pre and post-emergence stages.

In the same regard, results in Table (3) followed the same trends as in Table (2). All seed soaking treatments significantly reduce Fusarium root rot disease incidence on bean plants at pre and post-emergence stages when compared with control treatment. Combined treatments as well as commercial fungicide seed dressing treatment were the most effective in decreasing Fusarium root rot incidence compared with single or control treatments. The highest percentages of disease reduction were recorded with commercial fungicide and combined treatments of $\mathrm{CH} 1.0 \mathrm{~g} / \mathrm{l}+\mathrm{SA}$ or HA at rate of $5.0 \%$, the reduction percentages reached to 65.6 and $64.2 \%$, 65.4 and $60.7 \%$ and 63.5 and $57.8 \%$ for pre and postemergence stages, respectively. While, the percentages of plant survival were recorded by commercial fungicide (75\%) and combined treatments of $\mathrm{CH} 1.0 \mathrm{~g} / \mathrm{l}+\mathrm{SA} 5.0 \%$ (73.5) followed by SA $2.5 \%+$ HA $2.5 \%$ (72.6). The lowest concentrations of combined and single treatments showed less effect in controlling of root rot at pre and post-emergence stages.

These results are in agreement with those reported by many researchers, they used chemical resistance inducers for controlling root rot and wilt diseases under greenhouse and open field conditions (Abdel-Kareem, 1998). They have been demonstrated the potential of plant resistance inducers in controlling of damping-off and root rot diseases caused by $R$. solani and F. spp of many crops (Lewis and Lumsden, 2001; Abdel-Kareem, 2007; Yigit and Dikilitas, 2008; ElMohamedy and Ahmed, 2009; Bakeer, 2014). Plant chemical inducers might be stimulated some defense mechanisms such as phenolic compounds, oxidative enzymes and other metabolites (Amel et al., 2010; Abdel-Monaim et al., 2011; El-Mohamedy et al., 2013). In additions, some chemical inducers may also have a direct antimicrobial effect and are consequently involved in cross linking in cell walls, induction of gene expression, phyto-alexin production and induction of systemic resistance against plant diseases (El-Khallal, 2007; Akram and Anjum, 2011). Plants respond to chemical elicitor treatments by activating a wide variety of protective mechanisms designed to prevent pathogen replication and spreading (Terry and Joyce, 2004). The defense mechanisms include the fast production of reactive oxygen species (DeGara et al., 2003), alterations in the cell wall constitution; accumulation of antimicrobial secondary metabolites known as phyto-alexins (Anderson, 1988) and activation and/or synthesis of defense peptides and proteins, pathogenesisrelated proteins (Castro and Fontes, 2005).

\section{Control of root rot disease of green bean under open field conditions}

The efficacy of seed soaking followed by foliar application of plant resistance inducers (PRIs) as single or combined treatments against the incidence of damping-off and root rot diseases of green bean plants at pre (10 days) and post (40 and 60 days after sowing) emergence stages were evaluated under open field conditions during seasons of 2014/2015 and 2015/2016. Data presented in Table (4) clearly demonstrated that all applied treatments significantly reduced damping-off and root rot diseases in bean plants if compared with control treatment. Combined treatments of $\mathrm{CH}+\mathrm{SA}$ or HA were more effective in reducing damping-off and root rot diseases than single treatments and control treatment. These combined treatments resulted in the highest percentages of reduction in damping-off and root rot in both seasons. Soaking green bean seeds in $\mathrm{CH}$ at rate of $1 \mathrm{~g} / \mathrm{l}+\mathrm{SA}$ at $5 \%$ followed by spraying plants with half of this concentrations $(\mathrm{CH} 0.5 \mathrm{~g} / \mathrm{l}+\mathrm{SA}$ $2.5 \%$ ) resulted in the lowest values of infection and the highest percentages of reduction of damping-off and root rot incidence at all sampling dates $(10,40$ and 60 days after emergence), followed by a combined treatment of $\mathrm{CH}$ at rate of $1 \mathrm{~g} / \mathrm{l}+\mathrm{HA}$ at $5 \%$ and then sprayed plants with half of this concentrations $(\mathrm{CH} 0.5 \mathrm{~g} / \mathrm{l}+\mathrm{HA} 2.5 \%)$. Furthermore, the combined treatments $\mathrm{CH}+\mathrm{SA}$ or HA gave the highest percentages of plant survival when compared with other treatments during both seasons. It is of interest to note that such combined treatments were superior than commercial fungicide seed treatment in controlling damping-off and root rot diseases. Whereas, commercial fungicide (Rizolex-T) treatment recorded the infection values of $6.5,8.7,8.7$ and 6.4, 8.4, 8.2 at 10, 40 and 60 days after emergence in the first and second seasons, respectively. Also reduced damping-off and root rot incidence up to $64.6,60.4,64.0 \%$ and 64.0, 59.6, $63.4 \%$ at 10,40 and 60 days after emergence in the first and second seasons, respectively. Concerning the plant survival percentages fungicide treatment recorded 75.3 and $76.2 \%$ in the first and second seasons, respectively. 
Table 4. Effect of different chemical plant resistance inducers, chitosan, salicylic acid, humic acid and their combinations on damping-off and root rot diseases incidence of green bean plants grown under open field conditions during seasons of 2014/2015 and 2015/2016.

\begin{tabular}{|c|c|c|c|c|c|c|c|c|c|}
\hline \multirow{3}{*}{ Plant resistance inducers } & \multicolumn{2}{|c|}{ Treatments/concentrations } & \multirow{2}{*}{\multicolumn{2}{|c|}{$\begin{array}{c}\text { Pre-emergence } \\
\text { Damping-off after10 days }\end{array}$}} & \multirow{2}{*}{\multicolumn{2}{|c|}{$\begin{array}{l}\text { Root rot incidence } \\
\text { after } 40 \text { days }\end{array}$}} & \multirow{2}{*}{\multicolumn{2}{|c|}{$\begin{array}{l}\text { Root rot incidence } \\
\text { after } 60 \text { days }\end{array}$}} & \multirow{3}{*}{$\begin{array}{c}\text { Survival } \\
\text { plants } \\
\%\end{array}$} \\
\hline & \multirow{2}{*}{$\begin{array}{l}\text { Seed } \\
\text { soaking }\end{array}$} & \multirow{2}{*}{$\begin{array}{l}\text { Foliar } \\
\text { spraying }\end{array}$} & & & & & & & \\
\hline & & & Infection & $\begin{array}{c}\text { Disease } \\
\text { Reduction \% }\end{array}$ & Infection & $\begin{array}{c}\text { Disease } \\
\text { reduction \% }\end{array}$ & Infection & $\begin{array}{c}\text { Disease } \\
\text { reduction \% }\end{array}$ & \\
\hline First season 2014/2015 & & & & & & & & & \\
\hline Chitosan (CH) & $1.0 \mathrm{~g} / 1$ & $0.5 \mathrm{~g} / 1$ & $7.5 \mathrm{~d}$ & 59.0 & $10.5 \mathrm{~b}$ & 52.0 & $10.2 \mathrm{~b}$ & 57.8 & $71.8 \mathrm{~b}$ \\
\hline Salicylic acid (SA) & $5 \%$ & $2.5 \%$ & $8.8 \mathrm{~b}$ & 52.0 & $11.2 \mathrm{~b}$ & 49.2 & $11.6 \mathrm{~b}$ & 52.2 & $68.4 \mathrm{~b}$ \\
\hline Humic acid (HA) & & $2.5 \%$ & $9.5 \mathrm{~b}$ & 48.2 & $11.9 \mathrm{~b}$ & 46.0 & $12.1 \mathrm{~b}$ & 49.8 & $66.5 \mathrm{~b}$ \\
\hline $\mathrm{CH}+\mathrm{SA}$ & $1.0 \mathrm{~g} / 1+5 \%$ & $0.5 \mathrm{~g} / 1+2.5 \%$ & $5.8 \mathrm{~d}$ & 68.2 & $7.9 \mathrm{c}$ & 63.8 & $7.2 \mathrm{c}$ & 70.0 & $79.1 \mathrm{~b}$ \\
\hline $\mathrm{CH}+\mathrm{HA}$ & $1.0 \mathrm{~g} / 1+5 \%$ & $0.5 \mathrm{~g} / 1+2.5 \%$ & $6.3 \mathrm{~d}$ & 64.4 & $8.6 \mathrm{c}$ & 61.0 & $8.8 \mathrm{c}$ & 63.8 & $76.1 \mathrm{c}$ \\
\hline $\mathrm{SA}+\mathrm{HA}$ & $5+5 \%$ & $2.5+2.5 \%$ & $6.8 \mathrm{~d}$ & 63.0 & $9.2 \mathrm{c}$ & 58.2 & $9.4 \mathrm{c}$ & 61.2 & $75.7 \mathrm{c}$ \\
\hline Fungicide (Rhizolex-T 3g/kg seeds) & & & $6.5 \mathrm{~d}$ & 64.6 & $8.7 \mathrm{c}$ & 60.4 & $8.7 \mathrm{c}$ & 64.0 & $75.3 \mathrm{c}$ \\
\hline Control & & & $18.4 \mathrm{a}$ & 0.0 & $22.0 \mathrm{a}$ & 0.0 & $24.2 \mathrm{a}$ & 0.0 & $35.4 \mathrm{a}$ \\
\hline Second season 2015/2016 & & & & & & & & & \\
\hline Chitosan $(\mathrm{CH})$ & $1.0 \mathrm{~g} / 1$ & $0.5 \mathrm{~g} / 1$ & $7.2 \mathrm{~b}$ & 59.6 & $9.8 \mathrm{~b}$ & 52.9 & $9.4 \mathrm{~b}$ & 58.0 & $73.6 \mathrm{~b}$ \\
\hline Salicylic acid (SA) & $5 \%$ & $2.5 \%$ & $8.6 \mathrm{~b}$ & 51.7 & $10.6 \mathrm{~b}$ & 49.0 & $10.8 \mathrm{~b}$ & 51.8 & $70.0 \mathrm{~b}$ \\
\hline Humic acid (HA) & $5 \%$ & $2.5 \%$ & $9.2 \mathrm{~b}$ & 48.3 & $11.4 \mathrm{~b}$ & 45.2 & $11.4 \mathrm{~b}$ & 50.0 & $68.0 \mathrm{~b}$ \\
\hline $\mathrm{CH}+\mathrm{SA}$ & $1.0 \mathrm{~g} / 1+5 \%$ & $0.5 \mathrm{~g} / 1+2.5 \%$ & $5.8 \mathrm{c}$ & 67.4 & $7.6 \mathrm{c}$ & 63.3 & $6.8 \mathrm{c}$ & 69.6 & $79.8 \mathrm{c}$ \\
\hline $\mathrm{CH}+\mathrm{HA}$ & $1.0 \mathrm{~g} / 1+5 \%$ & $0.5 \mathrm{~g} / 1+2.5 \%$ & $6.0 \mathrm{c}$ & 66.3 & $8.8 \mathrm{c}$ & 57.7 & $8.2 \mathrm{c}$ & 63.3 & $77.0 \mathrm{c}$ \\
\hline $\mathrm{SA}+\mathrm{HA}$ & $5+5 \%$ & $2.5+2.5 \%$ & $6.4 \mathrm{c}$ & 64.0 & $8.4 \mathrm{c}$ & 59.6 & $9.0 \mathrm{c}$ & 59.8 & $76.2 \mathrm{c}$ \\
\hline Fungicide (Rhizolex-T 3g/kg seeds) & & & $6.4 \mathrm{c}$ & 64.0 & $8.4 \mathrm{c}$ & 59.6 & $8.2 \mathrm{c}$ & 63.4 & $76.2 \mathrm{c}$ \\
\hline 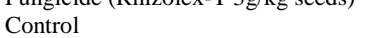 & & & $17.8 \mathrm{a}$ & 0.0 & $20.8 \mathrm{a}$ & 0.0 & $22.4 \mathrm{a}$ & 0.0 & $39.0 \mathrm{a}$ \\
\hline
\end{tabular}

Table 5. Effect of different chemical plant resistance inducers, chitosan, salicylic acid, humic acid and their combinations on vegetative growth characters of green bean plants grown under open field conditions during seasons of 2014/2015 and 2015/2016.

\begin{tabular}{|c|c|c|c|c|c|c|c|c|c|c|}
\hline \multirow{2}{*}{ Plant resistance inducers } & \multicolumn{2}{|c|}{ Treatments/concentrations } & \multirow{2}{*}{$\begin{array}{l}\text { Plant length } \\
(\mathrm{cm})\end{array}$} & \multicolumn{2}{|c|}{ Number of } & \multicolumn{2}{|c|}{ Fresh weight $(\mathrm{g})$} & \multicolumn{2}{|c|}{ Dry weight (g) } & \multirow{2}{*}{$\begin{array}{l}\text { Leaf area/plant } \\
\qquad\left(\mathrm{cm}^{2}\right)\end{array}$} \\
\hline & Seed soaking & Foliar spraying & & Leaves & Stems & Leaves & Stems & Leaves & Stems & \\
\hline \multicolumn{11}{|l|}{ First season 2014/2015 } \\
\hline $\mathrm{CH}$ & $1.0 \mathrm{~g} / \mathrm{l}$ & $0.5 \mathrm{~g} / 1$ & 42.67 & 8.57 & 5.51 & 13.10 & 12.67 & 5.16 & 5.98 & 114.53 \\
\hline SA & $5 \%$ & $2.5 \%$ & 44.52 & 8.44 & 6.35 & 13.17 & 13.47 & 5.24 & 6.00 & 114.75 \\
\hline HA & $5 \%$ & $2.5 \%$ & 45.56 & 8.87 & 6.34 & 14.17 & 14.83 & 5.44 & 6.28 & 114.89 \\
\hline $\mathrm{CH}+\mathrm{SA}$ & $1.0 \mathrm{~g} / 1+5 \%$ & $0.5 \mathrm{~g} / 1+2.5 \%$ & 51.20 & 10.96 & 7.60 & 17.40 & 17.93 & 7.21 & 8.01 & 150.40 \\
\hline $\mathrm{CH}+\mathrm{HA}$ & $1.0 \mathrm{~g} / 1+5 \%$ & $0.5 \mathrm{~g} / 1+2.5 \%$ & 49.25 & 10.55 & 7.51 & 16.70 & 17.03 & 6.34 & 7.46 & 145.53 \\
\hline $\mathrm{SA}+\mathrm{HA}$ & $5+5 \%$ & $2.5+2.5 \%$ & 49.11 & 10.43 & 7.41 & 16.55 & 16.92 & 6.09 & 7.33 & 133.98 \\
\hline Fungicide (Rhizolex-T 3g/kg seeds) & & & 50.01 & 10.51 & 7.53 & 16.78 & 17.00 & 6.38 & 7.62 & 148.13 \\
\hline Control & & & 39.07 & 7.99 & 5.18 & 11.64 & 10.40 & 4.50 & 5.00 & 111.41 \\
\hline LSD at 5\% & & & 4.73 & 1.06 & 1.42 & 2.75 & 2.88 & 1.23 & 1.09 & 7.17 \\
\hline \multicolumn{11}{|l|}{ Second season 2015/2016 } \\
\hline $\mathrm{CH}$ & $1.0 \mathrm{~g} / 1$ & $0.5 \mathrm{~g} / 1$ & 42.21 & 8.24 & 5.18 & 12.24 & 12.67 & 4.16 & 4.96 & 144.18 \\
\hline SA & $5 \%$ & $2.5 \%$ & 43.74 & 8.11 & 6.02 & 13.18 & 13.61 & 4.33 & 5.16 & 144.00 \\
\hline HA & $5 \%$ & $2.5 \%$ & 45.56 & 8.87 & 6.34 & 14.17 & 14.83 & 5.44 & 6.28 & 114.89 \\
\hline $\mathrm{CH}+\mathrm{SA}$ & $1.0 \mathrm{~g} / \mathrm{l}+5 \%$ & $0.5 \mathrm{~g} / 1+2.5 \%$ & 50.59 & 10.62 & 7.27 & 16.34 & 16.42 & 6.19 & 7.03 & 149.56 \\
\hline $\mathrm{CH}+\mathrm{HA}$ & $1.0 \mathrm{~g} / 1+5 \%$ & $0.5 \mathrm{~g} / 1+2.5 \%$ & 48.65 & 10.21 & 7.18 & 15.57 & 16.39 & 5.38 & 6.41 & 144.29 \\
\hline $\mathrm{SA}+\mathrm{HA}$ & $5+5 \%$ & $2.5+2.5 \%$ & 48.11 & 10.13 & 7.01 & 16.55 & 16.12 & 5.09 & 6.33 & 144.98 \\
\hline Fungicide (Rhizolex-T 3g/kg seeds) & & & 50.15 & 10.38 & 7.19 & 15.66 & 15.79 & 5.06 & 6.66 & 144.63 \\
\hline Control & & & 38.50 & 7.66 & 4.85 & 10.35 & 10.26 & 3.54 & 4.33 & 144.34 \\
\hline LSD at 5\% & & & 4.97 & 1.11 & 1.60 & 0.98 & 1.07 & 0.34 & 0.51 & 4.25 \\
\hline
\end{tabular}


Table 6. Effect of different chemical plant resistance inducers, chitosan, salicylic acid, humic acid and their combinations on yield characters of green bean plants grown under field conditions during seasons of 2014/2015 and 2015/2016.

\begin{tabular}{|c|c|c|c|c|c|c|c|c|c|}
\hline \multirow{2}{*}{$\begin{array}{l}\text { Plant } \\
\text { resistance } \\
\text { inducers }\end{array}$} & \multicolumn{2}{|c|}{ Treatments/concentrations } & \multirow{2}{*}{$\begin{array}{l}\text { No. } \\
\text { pods/ } \\
\text { plant }\end{array}$} & \multirow{2}{*}{ of } & \multirow{2}{*}{$\begin{array}{l}\text { Weight of } \\
\text { pods/plant } \\
\text { (g) }\end{array}$} & \multirow{2}{*}{$\begin{array}{l}\text { Average } \\
\text { weight } \\
\text { pod }(g)\end{array}$} & \multirow[b]{2}{*}{ of } & \multirow{2}{*}{$\begin{array}{l}\text { Total yield } \\
\text { of pods } / \mathrm{m}^{2} \\
(\mathrm{~kg})\end{array}$} & \multirow{2}{*}{$\begin{array}{l}\text { Total yield } \\
\text { of pods/fed. } \\
(\mathrm{kg})\end{array}$} \\
\hline & $\begin{array}{l}\text { Seed } \\
\text { soaking }\end{array}$ & $\begin{array}{l}\text { Foliar } \\
\text { spraying }\end{array}$ & & & & & & & \\
\hline \multicolumn{10}{|c|}{ First season 2014/2015 } \\
\hline SA & $5 \%$ & $2.5 \%$ & 17.74 & & 44.32 & 1.89 & & 9.22 & 3567.67 \\
\hline HA & $5 \%$ & $2.5 \%$ & 17.33 & & 44.35 & 1.93 & & 9.01 & 3568.33 \\
\hline $\mathrm{CH}+\mathrm{SA}$ & $1.0 \mathrm{~g} / 1+5 \%$ & $0.5 \mathrm{~g} / 1+2.5 \%$ & 21.17 & & 48.18 & 2.03 & & 9.87 & 3670.67 \\
\hline $\mathrm{SA}+\mathrm{HA}$ & $5+5 \%$ & $2.5+2.5 \%$ & 20.12 & & 46.03 & 2.00 & & 9.55 & 3645.75 \\
\hline \multirow{2}{*}{\multicolumn{3}{|c|}{ Fungicide (Rhizolex-T 3g/kg seeds) }} & 20.92 & & 47.09 & 1.98 & & 9.95 & 3689.33 \\
\hline & & & 16.37 & & 41.84 & 1.87 & & 8.76 & 3488.33 \\
\hline \multicolumn{3}{|c|}{ LSD at $5 \%$} & 2.51 & & 5.43 & 0.20 & & 1.07 & 181.41 \\
\hline \multicolumn{10}{|c|}{ Second season $2015 / 2016$} \\
\hline $\mathrm{CH}$ & $1.0 \mathrm{~g} / \mathrm{l}$ & $0.5 \mathrm{~g} / 1$ & 16.28 & & 33.61 & 1.52 & & 8.31 & 3463.67 \\
\hline SA & $5 \%$ & $2.5 \%$ & 16.69 & & 34.48 & 1.42 & & 8.42 & 3451.33 \\
\hline HA & $5 \%$ & $2.5 \%$ & 16.48 & & 34.45 & 1.80 & & 8.03 & 3459.67 \\
\hline $\mathrm{CH}+\mathrm{HA}$ & $1.0 \mathrm{~g} / \mathrm{l}+5 \%$ & $0.5 \mathrm{~g} / 1+2.5 \%$ & 19.86 & & 36.37 & 2.04 & & 8.77 & 3550.00 \\
\hline $\mathrm{SA}+\mathrm{HA}$ & $5+5 \%$ & $2.5+2.5 \%$ & 19.12 & & 36.03 & 2.00 & & 8.55 & 3545.75 \\
\hline \multicolumn{3}{|c|}{ Fungicide (Rhizolex-T 3g/kg seeds) } & 19.85 & & 37.36 & 1.90 & & 8.72 & 3570.33 \\
\hline \multicolumn{3}{|c|}{ Control } & 15.49 & & 31.46 & 1.06 & & 7.64 & 3362.67 \\
\hline \multicolumn{3}{|l|}{ LSD at $5 \%$} & 0.66 & & 1.02 & 0.32 & & 0.68 & 67.15 \\
\hline
\end{tabular}

Table 7. Effect of different chemical plant resistance inducers, chitosan, salicylic acid, humic acid and their combinations on chemical quality of green bean pods during the two seasons of 2014/2015 and 2015/2016.

\begin{tabular}{|c|c|c|c|c|c|c|c|}
\hline \multirow{2}{*}{$\begin{array}{l}\text { Plant resistance } \\
\text { inducers }\end{array}$} & \multicolumn{2}{|c|}{ Treatments/concentrations } & \multicolumn{5}{|l|}{$\%$} \\
\hline & $\begin{array}{l}\text { Seed } \\
\text { soaking }\end{array}$ & $\begin{array}{l}\text { Foliar } \\
\text { spraying }\end{array}$ & $\mathrm{N}$ & $\mathrm{P}$ & $\mathrm{K}$ & Protein & $\begin{array}{c}\text { Dry } \\
\text { matter }\end{array}$ \\
\hline \multicolumn{8}{|l|}{ First season $2014 / 2015$} \\
\hline $\mathrm{CH}$ & $1.0 \mathrm{~g} / \mathrm{l}$ & $0.5 \mathrm{~g} / 1$ & 3.13 & 0.344 & 2.12 & 19.56 & 6.65 \\
\hline SA & $5 \%$ & $2.5 \%$ & 3.12 & 0.344 & 2.14 & 19.66 & 6.60 \\
\hline $\mathrm{HA}$ & $5 \%$ & $2.5 \%$ & 3.16 & 0.345 & 2.14 & 19.56 & 6.77 \\
\hline $\mathrm{CH}+\mathrm{SA}$ & $1.0 \mathrm{~g} / \mathrm{l}+5 \%$ & $0.5 \mathrm{~g} / \mathrm{l}+2.5 \%$ & 3.17 & 0.342 & 2.17 & 19.79 & 7.14 \\
\hline $\mathrm{CH}+\mathrm{HA}$ & $1.0 \mathrm{~g} / \mathrm{l}+5 \%$ & $0.5 \mathrm{~g} / \mathrm{l}+2.5 \%$ & 3.15 & 0.348 & 2.16 & 19.85 & 7.16 \\
\hline $\mathrm{SA}+\mathrm{HA}$ & $5+5 \%$ & $2.5+2.5 \%$ & 3.07 & 0.345 & 2.10 & 19.44 & 7.09 \\
\hline \multicolumn{3}{|c|}{ Fungicide (Rhizolex-T 3g/kg seeds) } & 3.20 & 0.353 & 2.16 & 20.40 & 7.16 \\
\hline \multicolumn{3}{|c|}{ Control } & 3.03 & 0.339 & 2.07 & 19.38 & 6.31 \\
\hline \multicolumn{3}{|l|}{ LSD at 5\% } & 0.12 & 0.012 & 0.07 & 1.25 & 1.25 \\
\hline \multicolumn{8}{|c|}{ Second season 2015/2016 } \\
\hline $\mathrm{CH}$ & $1.0 \mathrm{~g} / \mathrm{l}$ & $0.5 \mathrm{~g} / \mathrm{l}$ & 3.10 & 0.303 & 2.13 & 18.09 & 7.13 \\
\hline SA & $5 \%$ & $2.5 \%$ & 3.13 & 0.301 & 2.12 & 18.12 & 7.10 \\
\hline $\mathrm{HA}$ & $5 \%$ & $2.5 \%$ & 3.12 & 0.322 & 2.14 & 19.13 & 7.12 \\
\hline $\mathrm{CH}+\mathrm{SA}$ & $1.0 \mathrm{~g} / \mathrm{l}+5 \%$ & $0.5 \mathrm{~g} / 1+2.5 \%$ & 3.17 & 0.331 & 2.18 & 19.51 & 7.15 \\
\hline $\mathrm{CH}+\mathrm{HA}$ & $1.0 \mathrm{~g} / \mathrm{l}+5 \%$ & $0.5 \mathrm{~g} / \mathrm{l}+2.5 \%$ & 3.16 & 0.333 & 2.17 & 19.62 & 7.17 \\
\hline $\mathrm{SA}+\mathrm{HA}$ & $5+5 \%$ & $2.5+2.5 \%$ & 3.13 & 0.330 & 2.16 & 19.32 & 7.14 \\
\hline \multicolumn{3}{|c|}{ Fungicide (Rhizolex-T 3g/kg seeds) } & 3.18 & 0.333 & 2.18 & 19.44 & 7.15 \\
\hline \multicolumn{3}{|c|}{ Control } & 3.00 & 0.290 & 2.05 & 17.28 & 6.99 \\
\hline \multicolumn{3}{|l|}{ LSD at $5 \%$} & 0.18 & 0.05 & 0.05 & 0.70 & 0.70 \\
\hline
\end{tabular}

Meanwhile, no significant differences were realized between the combined treatments of soaking green bean seeds in $\mathrm{CH}$ at $1.0 \mathrm{~g} / 1+\mathrm{SA}$ or HA at rate of $5.0 \%$ and then sprayed bean plants with the half of this concentrations $(\mathrm{CH} 0.5 \mathrm{~g} / \mathrm{l}+\mathrm{SA}$ or HA $2.5 \%$ ). these treatments resulted in a considerable effect in suppressing of root rot disease incidence $(68.2,64.4,63.8$, $61.0,70.0,63.8 \%$ in the first season and $67.4,66.3,63.3$, $57.7,69.6,63.3 \%$ in the second season), and recorded the lowest values for plant infection $(5.8,6.3,7.9,8.6,7.2,8.8$ in the first season and 5.8, 6.0, 7.6, 8.8, 6.8, 8.2 in the second season) for 10, 40 and 60 days after emergence, respectively, as well as the highest percentages of plant survival (79.1 and $76.1 \%$ in the first season and 79.8 and $77.0 \%$ in the second season) if compared with the rest treatments in both seasons. These obtained results are in accordance with those reported by Abd-El-Kareem (2007); Abdel-Monaim et al. (2011); ElMohamedy et al. (2013). In this regard, soaking sesame seeds in salicylic acid for $24 \mathrm{~h}$ before sowing followed by foliar application after 15 days from sowing resulted in the best control against $F$. oxysporum compared to fungicide Benlate
(Abdou et al., 2001). In addition, Shahda (2000) stated that using benzoic acid, salicylic acid and ascorbic acid significantly reduced damping-off, the reduction in disease incidence ranged from $36-74 \%$. The antioxidants used were superior to fungicide Rizolex- $\mathrm{T}$ in controlling damping-off disease. Also Mandel et al. (2009) added that exogenous or endogenous factors could substantially affect host physiology, leading to rapid and coordinated activation of defense-gene in plants expressing susceptibility to pathogen infection. However, Jabnoun et al. (2015) reported that salicylic acid and chitosan can be used as inducers of systemic acquired resistance for controlling tomato fungal diseases.

Effect of plant resistance inducers (PRIs) on vegetative growth of green bean plants

Data presented in Table (5) obviously indicated that combined treatments of plant resistance inducers significantly increased vegetative growth characters such as plant length, 
number of leaves and branches, fresh and dry weight of leaves and branches as well as leaf area/plant during two successive seasons of 2014/2015 and 2015/2016 compared with the rest of treatments. The highest values of vegetative growth parameters were recorded by combined treatments $\mathrm{CH}+\mathrm{SA}, \mathrm{CH}+\mathrm{HA}, \mathrm{SA}+\mathrm{HA}$ and commercial fungicide (Rizolex-T) without significant differences among them. While, the lowest values for vegetative growth parameters were obtained by control treatment. A significant difference were detected only between combined treatments and control treatment. Also it is noticed that combined treatments were more superior than single treatments. These findings were true in both seasons of study. These results are in harmony with those obtained by Khan et al. (2002) and Chibu and Shibayama (2003). They reported that application of chitosan increased key enzymes activities of nitrogen metabolism and improved the transportation of nitrogen in the functional leaves which enhanced plant growth and development. Foliar applications with chitosan resulted in better vegetative growth and improvement of fruit quality of tomato, pepper, radish, cucumber and okra (Farouk et al., 2008; Mondal et al., 2012; Shehata et al., 2012; Abd El-Gawad and Bondok, 2015). However, Sheikha and Al-Malki (2011) found that chitosan treatment enhanced bean shoot and root length, fresh and dry weights of leaves, shoots and root. A significance role of chitosan as plant growth promoting and disease control agents has been emphasized (Trotel-Aziz et al., 2006).

\section{Effect of plant resistance inducers on yield characters of green bean plants}

The results in Table (6) strongly revealed that combined treatments were more efficient than single treatments in terms of green bean yield and its characters. Combined treatments of $\mathrm{CH}+\mathrm{SA}(1.0 \mathrm{~g} / \mathrm{l}+5 \%), \mathrm{CH}+\mathrm{HA}(1.0 \mathrm{~g} / \mathrm{l}+5 \%)$ and $\mathrm{SA}$ $+\mathrm{HA}(5+5 \%)$ as well as commercial fungicide treatment recorded the highest values of all determined characters i.e. average weight of pod, number of pods/plant, weight of pods/plant, total yield of pods $/ \mathrm{m}^{2}$ and total yield of pods/fed. without significant differences among them in both seasons of study. Whereas, the lowest values of all determined characters were detected with control treatment in both seasons. It is of interest to notice that during both seasons, no significant differences were realized among single treatments, similarly, among combined treatments. A significant difference was detected only between combined treatments and control treatment. The trends of obtained results are in good accordance with Akinci et al. (2009) they concluded that humic acid contains many elements which improve the soil fertility and increase the availability of nutrient elements consequently affect the yield and significantly increased weight of green pod yield of snap bean (El-Bassiony et al., 2010) and hot pepper yield (Khalil et al., 2012).

\section{Effect of plant resistance inducers on chemical quality of green bean pods}

Data shown in Table (7) clearly revealed that commercial fungicide treatment (Rizolex-T) significantly increased nitrogen, phosphorus, protein contents and percentage of dry matter in pods of green bean plants in the first season and nitrogen, phosphorus as well as potassium contents in the second season if compared with single and control treatments. On the other hand, combined treatment of $\mathrm{CH} 1.0$ $\mathrm{g} / \mathrm{l}+\mathrm{SA} 5 \%$ gave the best value of potassium percentage in the first season, however, combined treatment of $\mathrm{CH} 1.0 \mathrm{~g} / 1+$
HA 5\% in the second season gave the best values of protein and dry matter percentages. The highest values of determined chemical quality parameters of green bean pods were fluctuated among commercial fungicide, $\mathrm{CH}+\mathrm{HA}$ and $\mathrm{CH}+$ SA treatments without significant differences among them in both seasons of 2014/2015 and 2015/2016, while, control treatment gave the lowest values of chemical quality parameters. In spite of, no significant differences were detected among combined and commercial fungicide treatments as well as among single treatments and control treatment, significant differences were noticed only between commercial fungicide treatment, combined treatments and control treatment on most of measured characters in both seasons. The obtained results are in good accordance with Shehata et al. (2012); Abd El-Gawad and Bondok (2015); ElMohamedy et al. (2015). They reported that application of inducer resistance chemicals have been proved to increase yield and quality. Application of humic acid enhances nutrient uptake and quality as well in a number of plant species (Zaky et al., 2006; Yildirim, 2007; Karakurt et al., 2009). Moreover, it led to increase the percentage nitrogen and protein contents as well as Fe content in broad bean seeds (Shafeek et al., 2013).

\section{Materials and Methods}

\section{Source of green bean seeds and pathogenic fungi}

Green bean seeds cv. Giza 3 were kindly obtained from Vegetable Crops Research Dept., Horticultural Research Institute (HRI), Agricultural Research Center (ARC), Giza, Egypt. Fusarium solani and Rhizoctonia solani were isolated from green bean field showed typical root rot disease symptoms such as root and hypocotyl rot, damping off, stunted, yellowed, wilted and defoliation plants and the most diagnostic symptom is reddish-brown streaks within the vascular tissue up to and beyond the first node (ElMohamedy et al., 2013).

Effect of plant resistance inducers (PRIs) on growth of $F$. solani and $R$. solani

The inhibitory effect of different plant resistance inducers (PRIs) such as chitosan (CH), salicylic acid (SA) and humic acid (HA) against $F$. solani and $R$. solani the causal agent of root rot diseases of green bean was tested in vitro. Different concentrations of chitosan $0.25,0.5,1.0$ and $2.0 \mathrm{~g} / \mathrm{l}$; salicylic acid $1.25,2.5$ and $5.0 \%(\mathrm{w} / \mathrm{v})$ and humic acid $1.25,2.5$ and $5.0 \%(\mathrm{w} / \mathrm{v})$ were added individually or in combination to the sterilized PDA medium before solidifying and gently rotating and disbanding into sterilized Petri plates $(9 \mathrm{~cm}$ diameter). Plates were individually inoculated at the center with equal disks (6 $\mathrm{mm}$ in diameter) taken from eight days old cultures of each $F$. solani and $R$. solani, then all inoculated plates were incubated at $25 \pm 2^{\circ} \mathrm{C}$. Each treatment was represented by 4 plates and considered as replicates. Linear growth of tested fungi was measured when the control plates (medium free of chemical inducers) reached to full growth and the average growth diameter was calculated.

Control of root rot pathogens of green bean under greenhouse conditions

This experiment was carried out to evaluate the efficiency of different plant resistance inducers in controlling of root rot diseases caused by $F$. solani and $R$. solani on green bean 
plants grown in artificially infested soil under greenhouse. The applied treatments were as follows:

$1-\mathrm{CH}$ at $1.0 \mathrm{~g} / \mathrm{l}$ as seed soaking followed by $\mathrm{CH}$ at $0.5 \mathrm{~g} / \mathrm{l}$ as foliar spraying;

2- SA at $2.5 \%$ as seed soaking followed by SA at $1.25 \%$ as foliar spraying;

3- SA at $5.0 \%$ as seed soaking followed by SA at $2.5 \%$ as foliar spraying;

4- $\mathrm{HA}$ at $2.5 \%$ as seed soaking followed by $\mathrm{HA}$ at $1.25 \%$ as foliar spraying;

5- $\mathrm{HA}$ at $5.0 \%$ as seed soaking followed by $\mathrm{HA}$ at $2.5 \%$ as foliar spraying;

6- $\mathrm{CH}$ at $1.0 \mathrm{~g} / \mathrm{l}+\mathrm{SA}$ at $2.5 \%$ as seed soaking followed by $\mathrm{CH}$ at $0.5 \mathrm{~g} / \mathrm{l}+\mathrm{SA}$ at $1.25 \%$ as foliar spraying;

7- $\mathrm{CH}$ at $1.0 \mathrm{~g} / \mathrm{l}+\mathrm{SA}$ at $5.0 \%$ as seed soaking followed by $\mathrm{CH}$ at $0.5 \mathrm{~g} / \mathrm{l}+\mathrm{SA}$ at $2.5 \%$ as foliar spraying;

8- $\mathrm{CH}$ at $1.0 \mathrm{~g} / \mathrm{l}+\mathrm{HA}$ at $2.5 \%$ as seed soaking followed by $\mathrm{CH}$ at $0.5 \mathrm{~g} / \mathrm{l}+\mathrm{HA}$ at 1.25 as foliar spraying;

9- $\mathrm{CH}$ at $1.0 \mathrm{~g} / \mathrm{l}+\mathrm{HA}$ at $5.0 \%$ as seed soaking followed by $\mathrm{CH}$ at $0.5 \mathrm{~g} / \mathrm{l}+\mathrm{HA}$ at $2.5 \%$ as foliar spraying;

$10-\mathrm{SA}$ at $2.5 \%+\mathrm{HA}$ at $2.5 \%$ as seed soaking followed by SA at $1.25 \%+\mathrm{HA}$ at $1.25 \%$ as foliar spraying;

$11-\mathrm{SA}$ at $5.0 \%+\mathrm{HA}$ at $5.0 \%$ as seed soaking followed by $\mathrm{SA}$ at $2.5 \%+\mathrm{HA}$ at $2.5 \%$ as foliar spraying;

12- Commercial fungicide treatment (Rizolex-T 50\% at rate of $3 \mathrm{~g} / \mathrm{kg}$ seeds for seed treating);

13- Control treatment (untreated seeds).

Green bean seeds cv. Giza 3 were soaked for 24 hours before sowing in chemical inducers as previously explained and then sown in plastic pots $(25 \mathrm{~cm}$ diam.) filled with artificially infested loamy soil by inocula of either $F$. solani or $R$. solani at rate of $5 \%$ of soil weight (Abdel-Kader, 1997). Comparison treatment included a set of green bean seeds treated with commercial fungicide Rizolex-T and untreated seeds (control treatment) were also sown in plastic pots. Five seeds were sown in each pot, and ten pots were considered as a replicate for each particular treatment, the experiment design was a complete randomized design. Foliar application with half concentrations of used rate for seed soaking treatment was applied for three times in 7 days interval starting from the second true leaf stage of emerged bean seedlings.

The percentage of root rot incidence of bean at pre and post-emergence stages were calculated after 10 days and up to 40 days of the experimental period, respectively. Preemergence percentage was calculated based on the number of un-emerged seeds in relation to the number of sown seeds, while post-emergence percentage was calculated based on the number of plants showing disease symptoms in relation to the number of emerged seedlings.

\section{Control of root rot diseases of green bean plants under open} field conditions

Field experiments were carried out during seasons of 2014/2015 and 2015/2016 at the Experimental Research Station of National Research Centre at El-Noubaria region, Behera Governorate, Egypt. This field is well known by the authors as naturally heavily infested with soil borne pathogens. The efficacy of the most promising treatments obtained from greenhouse experiment for controlling root rot diseases in bean were applied under field conditions. The commercial fungicide Rizolex-T was used as a comparison to the other treatments. The evaluated treatments applied were as follows:

$1-\mathrm{CH}$ at $1.0 \mathrm{~g} / \mathrm{l}$ as seed soaking followed by $\mathrm{CH}$ at $0.5 \mathrm{~g} / \mathrm{l}$ as foliar spraying;
2- SA at $5.0 \%$ as seed soaking followed by SA at $2.5 \%$ as foliar spraying;

3- $\mathrm{HA}$ at $5.0 \%$ as seed soaking followed by $\mathrm{HA}$ at $2.5 \%$ as foliar spraying;

4- $\mathrm{CH}+\mathrm{SA}$ at $1.0 \mathrm{~g} / \mathrm{l}+5.0 \%$ as seed soaking followed by $\mathrm{CH}+\mathrm{SA}$ at $0.5 \mathrm{~g} / \mathrm{l}+2.5 \%$ as foliar spraying.

$5-\mathrm{CH}+\mathrm{HA}$ at $1.0 \mathrm{~g} / \mathrm{l}+5.0 \%$ as seed soaking followed by $\mathrm{CH}+\mathrm{HA}$ at $0.5 \mathrm{~g} / \mathrm{l}+2.5 \%$ as foliar spraying.

6- SA + HA at $5.0 \%+5.0 \%$ as seed soaking followed by SA $+\mathrm{HA}$ at $2.5 \%+2.5 \%$ as foliar spraying.

7- Commercial fungicide treatment (Rizolex-T $50 \%$ at rate of $3 \mathrm{~g} / \mathrm{kg}$ seeds for seed treating, was used as a comparison treatment.

8- Control treatment (untreated seeds).

All seed treatments were applied in a complete randomized block design with five replicates (plots) for each particular treatment. The green bean seeds cv. Giza 3 were soaked for 24 hours in chemical inducers treatments as explained previously, then sown at rate of 3 seeds per hill and $30 \mathrm{~cm}$ distance between hills on one side of drip irrigated ridge with $70 \mathrm{~cm}$ width and $5 \mathrm{~m}$ length on the second week of September in both seasons of 2014/2015 and 2015/2016. Each experimental plot included 3 ridges with a net area of $10.5 \mathrm{~m}^{2}$. Foliar spraying with half concentrations of rate used for seed soaking treatment was applied for three times in 7 days interval starting from the second true leaf stage of emerged bean seedlings.

The percentage of root rot incidence at the pre-emergence stage was calculated as the number of absent emerged seedlings in relative to the total number of sown seeds. Meanwhile, the percentage of post-emergence root rot diseases was calculated as the number of bean plants showing disease symptoms in relative to the total number of emerged seedlings. The percentages of pre-emergence root rot incidence was calculated after 10 days from sowing date, meanwhile, bean root rot incidence was recorded periodically every 10 days starting from 30 up to 60 days after sowing during bean plants growth and development.

Effect of plant resistance inducers (PRIs) on vegetative growth and yield of green bean Plant vegetative growth measurements

A representative sample of ten plants was randomly taken 45 days after sowing (at flowering stage), from each experimental sub-plot for measuring the plant vegetative growth parameters i.e. plant length, number of leaves and branches per plant as well as fresh weight of leaves and stems and dry weight of leaves and stems as well as leaf area/plant.

\section{Green pod yield and pod quality parameters}

At harvesting stage (after 60 days from sowing), the total green pods from each experimental sub-plot were collected along the harvesting period (50 days) and the following data were recorded: average weight of pods per plant $(\mathrm{g})$, average number of pods per plant, total green pods yield as $\mathrm{kg} / \mathrm{m}^{2}$ and ton/fed. Moreover, a random sample of 25 green pods from the $3^{\text {rd }}$ and $4^{\text {th }}$ harvestings were taken from each experimental plots to record average pod weight $(\mathrm{g})$.

\section{Nutritional value of green bean pods}

A sample of 25 green pods from the $3^{\text {rd }}$ and $4^{\text {th }}$ harvestings was randomly taken and oven dried to determined the following parameters: nitrogen percentage in pod according to the method of Pregl (1945). In addition, protein percentage 
in pod was calculated by multiplying nitrogen percentage by 6.25. Potassium was assayed using flame spectrophotometer according to Allen et al. (1984). Phosphorous was extracted and measured using spectrophotometer according to Jackson (1965). As well as dry matter percentage was determined according to the method of Dubois et al. (1956).

\section{Statistical analysis}

All data obtained were subjected to statistical analysis using the analysis of variance with SPSS software. Mean of the treatments were separated using least significant differences (LSD) test at $(\mathrm{P} \leq 0.05)$ level of significance according to the procedures reported by Snedecor and Cochran (1989).

\section{Conclusion}

Results of the current study suggested that, combined treatments of chemical plant resistance inducers $(\mathrm{CH}+\mathrm{SA}$ or HA) were superior than single treatments application in inducing green bean plant resistance to soil-borne pathogens ( $F$. solani and $R$. solani) and improving growth, pods yield and quality. However, the combined treatments had the best effect than commercial fungicide treatment either in controlling the diseases or in promoting the plant growth and improving pods yield and quality. Such combined treatments might be commercially used as an eco-friendly, safe, cheap and easily applied method alternative to fungicides under open field conditions.

\section{Acknowledgement}

The authors would like to thank National Research Centre (NRC), Egypt for the support in conducting current study through in house grant (Safety production of some vegetable crops, ID-10120207), within the tenth research plan.

\section{References}

Abd El-Gawad HG, Bondok AM (2015) Response of tomato plants to salicylic acid and chitosan under infection with tomato mosaic virus. Am-Euras J Agric Environ Sci. 15(8): 1520-1529.

Abdel-Kader MM (1997) Field application of Trichoderma harzianum as biocide for control bean root rot disease. Egypt J Phytopath. 25: 19-25.

Abdel-Kareem FI (1998) Induction of resistance to some disease of cucumber plants grown under greenhouse condition. PhD Thesis Fac of Agric Ain Shams Univ. Cairo Egypt.

Abdel-Kareem FI (2007) Induced resistance in bean plants against root rot and Alternaria leaf spot diseases using biotic and abiotic inducers under field conditions. Res $\mathbf{J}$ Agric Biol Sci. 3(6): 767-774.

Abdel-Mawgoud AMR, Tantawy AS, El-Nemr MA, Sassine YN (2010) Growth and yield responses of strawberry plants to chitosan application. Euro J Sci Res. 39(1): 170-177.

Abdel-Monaim MF (2013) Improvement of bio control of damping-off and root rot/wilt of faba bean by salicylic acid and hydrogen peroxide. Plant Path Res Inst. Agric Res Center. Giza Egypt 41(1): 47-55.

Abdel-Monaim MF, Ismail ME, Morsy KM (2011) Induction of systematic resistance in soybean plants against Fusarium wilt disease by seed treatment with benzothiadiazole and humic acid. Not Sci Biol. 3(2): 80-89.

Abdel-Monaim MF, Abdel-Gaid MAW, Armanious HAH (2012) Effect of chemical inducers on root rot and wilt diseases, yield and quality of tomato. Int J Agric Sci. 2(7): 210-220.

Abdou El-S, Abd-Alla HM, Galal AA (2001) Survey of sesame root rot/wilt disease in Minia and their possible control by ascorbic and salicylic acids. Assuit J Agric Sci. 32(3): 135-152.

Akinci S, Buyukkeskin T, Eroglu A, Erdogan BE (2009) The effect of humic acid on nutrient composition in broad bean roots. Not Sci Biol. 1: 81-87.

Akram W, Anjum T (2011) Use of bio agents and synthetic chemicals for induction of systemic resistance in tomato against diseases. Int Res J Agric Sci Soil Sci. 1: 286-292.

Allen SF, Grimshaw HF, Rowl AB (1984) Chemical analysis. In: Moor PD, Chapman SB (Eds) Methods in Plant Ecology, Blackwell, Oxford, UK. 185-344.

Amel A, Soad H, Ahmed M, Ismail AA (2010) Activation of tomato plant defense response against Fusarium wilt disease using Trichoderma harzianum and salicylic acid under greenhouse conditions. Res J Agric Biol Sci. 6: 328338.

Anderson AJ (1988) Elicitors the hypersensitive response and phytoalexins. In: Keen NT, Kosuge T, Walling LL (Eds.) Physiology and Biochemistry of Plant-Microbial Interactions, Amer Soc Plant Physiol., Rockville, MD, USA. 103-110.

Bakeer AT (2014) Effect of combination between inducer resistance chemical and systemic fungicides on management of sweet melon downy mildew. Arch Phytopathology Plant Prot. 47(14): 1716-1725.

Castro MS, Fontes W (2005) Plant defense and antimicrobial peptides. Protein Peptide Lett. 12: 11-16.

Chen Y, De Nobili M, Aviad T (2004) Stimulatory effect of humic substances on plant growth. In: Magdoff F, Weil RR (Eds) Soil Organic Matter in Sustainable Agriculture, Press Boca Raton FL, USA. 103-130.

Chibu H, Shibayama H (2003) Effects of chitosan application on the growth of several crops. In: Uragami T, Kurita K, Fukamizo T (Eds) Chitin and Chitosan in Life Science, Yamaguchi, Japan. 235-239.

De-Gara L, de Pinto MC, Tommasi F (2003) The antioxidant systems vis-à-vis reactive oxygen species during plant pathogen interaction. Plant Physiol Biochem. 41: 863-870.

Dubois M, Gilles KA, Hamilton JK, Robors PA, Smith F (1956) Colorimetric method for determination of sugars and related substances. Anal Chem. 28(3): 350-356.

El-Bassiony AM, Fawzy ZF, Abd El-Baky MMH, Mahmoud AR (2010) Response of snap bean plants to mineral fertilizers and humic acid application. Res J Agric Biol Sci. 6(2): 169-175.

El-Khallal SM (2007) Induction and modulation of resistance in tomato plants against Fusarium wilt disease by bio agent fungi (Arbuscular mycorrhiza) and/or hormonal elicitors (jasmonic acid and salicylic acid): I- Changes in growth, some metabolic activities and endogenous hormones related to defense mechanism. Aust J Basic Appl Sci. 1: 691-705.

El-Mohamedy RSR, Ahmed MA (2009) Effect of biofertilizers and humic acid on control of dry root rot disease and improvement yield quality of mandarin (Citrus reticulate Blanco). Res J Agric Biol Sci. 5(2): 127-137.

El-Mohamedy RSR, Abdel-Kader MM, Abd El-Kareem FI, El-Mougy NS (2013) Inhibitory effect of antagonistic bioagents and chitosan on the growth of tomato root rot pathogens in vitro. J Agric Tech. 9(6): 1521-1533.

El-Mohamedy RSR, Shafeek MR, Shaheen AM, Abd ElSamad EH, Fatma A Rizk, Morsey AA (2015) Integration between seed bio priming and potassium salts treatments to 
control of root rot disease and improvement growth and yield of green bean plants. Curr Sci Int. 4(2): 155-163.

El-Mougy NS, Nadia G, Abdel-Kader MM (2007) Control of wilt and root rot incidence in Phaseolus vulgaris L. by some plant volatile compounds. J Plant Prot Res. 47(3): 255-265.

Farouk S, Ghoneem KM, Abeer AA (2008) Induction and expression of systematic resistance to downy mildew disease in cucumber plant by elicitors. Egypt $J$ Phytopathology. 1-2: 95-111.

Hilal AA, Nada MGA, Zaky MH (2006) Induced resistance against Sclerotinia sclertiorum disease in some Umbelliferous medicinal plants as a possible and effective control mean. Egypt J Phytopathology. 34: 85-101.

Jabnoun KH, El-Mohamedy RSR, Abdel-Kareem FI, Ben Abdallah RA, Gueddes-Chahed M, Daami-Remadi M (2015) Variation in chitosan and salicylic acid efficacy towards soil-borne and air-borne fungi and their suppressive effect of tomato wilt severity. J Plant Pathol Microbiol. 6(11): 1-10.

Jackson ML (1965) Soil chemical analysis advanced course. Wisconsin State Univ. Wisconsin, USA.

Karakurt Y, Unlu H, Padem H (2009) The influence of foliar and soil fertilization of humic acid on yield and quality of pepper. Acta Agric Scandinavica. 59(3): 233-237.

Khalil AM, Khalil SE, Ali TB (2012) Effect of water stress, antioxidants and humic acid on Capsicum annumm L. growth, yield and active ingredient under sandy soil conditions. Egypt J Appl Sci. 27(1): 35-56.

Khan WM, Prithiviraj B, Smiyh DL (2002) Effect of foliar application of chitin oligosaccharides on photosynthesis of maize and soybean. Photosynthetica. 40: 621-624.

Kim HJ (2005) Characterization of bioactive compounds in essential oils, fermented anchovy sauce, and edible plants, and, induction of phytochemicals from edible plants using methyl jasmonate (MeJA) and chitosan. Ph.D. Thesis Clemson Univ. USA.

Lewis JA, Lumsden RD (2001) Biocontrol of damping-off of greenhouse grown crops caused by Rhizoctonia solani with a formulation of Trichoderma spp. Crop Prot. 20: 49-56.

Mandel MJ, Wollenberg MS, Stabb EV, Visick KL, Ruby EG (2009) A single regulatory gene is sufficient alter bacterial host range. Nature. 458: 215-218.

Mondal MMA, Malek MA, Puteh AB, Ismail MR, Ashrafuzzaman M, Naher L (2012) Effect of foliar application of chitosan on growth and yield in okra. Aust $\mathbf{J}$ Crop Sci. 6(5): 918-921.

Pregl F (1945) Quantitative organic micro-analysis $1^{\text {st }}$ Ed. J \& A Churdill Ltd. London, UK.
Scheuerell SJ, Mahaffee WF (2004) Compost tea as a container medium drench for suppressing seedling damping-off caused by Pythium ultimum. Phytopathology. 94: 1156-1163.

Shafeek MR, Helmy YI, Nadia OM, Fatma A Rizk (2013) Effect of foliar fertilizer with nutritional compound and humic acid on growth and yield of broad bean plants under sandy soil conditions. J Appl Sci Res. 9(6): 3674-3680.

Shahda WT (2000) The use of antioxidants for control of tomato damping-off. Alexandria J Agric Res. 45(1): $307-$ 316.

Shehata SA, Fawzy ZF, El-Ramady HR (2012) Response of cucumber plants to foliar application of chitosan and yeast under greenhouse conditions. Aust J Basic Appl Sci. 6(4): 63-71.

Sheikha SA, Al-Malki FM (2011) Growth and chlorophyll responses of bean plants to chitosan applications. Eur J Sci Res. 50(1): 124-134.

Snedecor GW, Cochran WG (1989) Statistical methods. $8^{\text {th }}$ Ed. Iowa State Univ. Press, Ames Iowa, USA.

Terry LA, Joyce DC (2004) Elicitors of induced disease resistance in postharvest horticultural crops: a brief review. Postharvest Biol Tech. 32: 1-13.

Trotel-Aziz P, Couderchet M, Vernet G, Aziz A (2006) Chitosan stimulates defense reactions in grapevine leaves and inhibits development of Botrytis cinerea. Eur J Plant Pathol. 114: 405-413.

Yigit F, Dikilitas M (2008) Effect of humic acid application on the root-rot diseases caused by Fusarium spp. on tomato plants. Plant Pathol J. 7(2): 179-182.

Yildirim E (2007) Foliar and soil fertilization of humic acid affect productivity and quality of tomato. Acta Agric Scand Sect B Soil Plant Sci. 57: 182-186.

Zaky MH, El-Zeiny OAH, Ahmed ME (2006) Effects of humic acid on growth and productivity of bean plants grown under plastic low tunnels and open field. Egypt J Appl Sci. 21(4B): 582-596. 